\title{
Differences of fatty acid composition in Brazilian genetic and conventional soybeans (Glycine max (L.) Merrill) grown in different regions
}

\author{
Olívio Fernandes Galão ${ }^{a}$, Mercedes Concórdia Carrão-Panizzi ${ }^{a}$, José Marcos Gontijo Mandarino ${ }^{\text {b }}$, \\ Oscar Oliveira Santos Júnior ${ }^{c}$, Swami Arêa Maruyama ${ }^{c}$, Luana Caroline Figueiredo ${ }^{\mathrm{d}}$, \\ Elton Guntendorfer Bonafe ${ }^{\mathrm{d}, *}$, Jesui Vergilio Visentainer ${ }^{\mathrm{c}}$ \\ a Departamento de química, Universidade Estadual de Londrina Rodovia Celso Garcia Cid, PR 445, Km 380, Campus Universitário Cx. Postal 6001 CEP 86055-900 Londrina, PR, Brazil \\ b EMBRAPA-Centro Nacional de Pesquisa de Soja (CNPSo), Caixa Postal 231, CEP 86001-970 Londrina, PR, Brazil \\ c Departamento de Química, Universidade Estadual de Maringá, Av. Colombo, 5790, CEP 87020-900, Maringá, Paraná, Brazil \\ d Departamento de química, Universidade Tecnológica Federal do Paraná, Rua Marcílio Dias, 635, CEP 86812-460, Apucarana, Paraná, Brazil
}

\section{A R T I C L E I N F O}

\section{Article history:}

Received 11 February 2014

Accepted 13 April 2014

Available online 24 April 2014

\section{Keywords:}

Soybeans

Fatty acids

Transgenic soybeans

Geographic location

\begin{abstract}
A B S T R A C T
The aim of this study was to compare fatty acid profiles of seed samples from twenty different soybean (Glycine $\max (\mathrm{L}$.) Merrill) genotypes (14 non-transgenic and six transgenic Roundup-tolerant) grown at two different locations, both in the Parana state, a southern region of Brazil. A total of eleven fatty acids were detected and quantified, among them the most expressive ones were oleic, linoleic, linolenic and palmitic acids. The total unsaturated fatty acid amount was higher than $82 \%$. An increase in the n-3 fatty acids quantities were observed in transgenic species, which can be reflected in lower n-6/n-3 ratios, a highly desired trend regarding consumers' health. In conclusion, results showed a large amount of variation among the different germplasms (either conventional or transgenic) within and across locations.
\end{abstract}

(c) 2014 Elsevier Ltd. All rights reserved.

\section{Introduction}

Soybean (Glycine max (L.) Merrill) is the most important oilseed of worldwide interest (Cheng et al., 2008; EMBRAPA, 2011 and Lee et al., 2008) and Brazil has become one of its largest producers (Berman et al., 2010). The oil which is derived from it possesses a fundamental role in the diet of many South American people. The majority of soybean cultivars present around $15-20 \%$ of lipidic fraction, and this value is influenced by climate conditions, geographical localization, soil and selected agronomic procedures (Kumar, Rani, Solanki, \& Hussain, 2006; Souza, Zanon, Pedroso, \& Andrade, 2009).

Transgenic soy line GTS-40-3-2, more commonly known as roundup ready (RR) soybeans, was developed by Monsanto (USA) to allow the use of glyphosate, the active ingredient of herbicide Roundup as a weed control agent. RR beans were first approved in Canada for environmental release and for feed products in 1995 (Lerat et al., 2005; Rott, Lawrence, Wall, \& Green, 2004). Droste, Pasquali, and BodaneseZanettini (2002) describes the production of transgenic and fertile soybean plants (Glycine max (L.) Merrill): the transformation method combines the advantages of somatic embryogenesis with the efficiency of

Abbreviations: RR, roundup ready; LNA, linolenic acid; n-3, omega 3 fatty acids; n-6, omega 6 fatty acids; LC, long chain; PUFA, polyunsaturated fatty acids; FAME, fatty acids methyl ester; PCA, principal component analysis.

* Corresponding author. Tel.: +55 4488080350.

E-mail address: eltongbonafe@gmail.com (E.G. Bonafe). particle bombardment of tissues that have great in vitro proliferation and regeneration capacities. This article first described the results for transformation of soybean cultivars recommended for commercial production in southern Brazilian regions by using somatic embryogenesis.

The overall composition of fatty acids and other bioactive compounds in soy are determined by its genotype (Lee et al., 2010; Maestri et al., 1998). Soy samples with altered fatty acid composition have been developed through mutation breeding (Patil, Taware, Oak, Tamhankar, \& Rao, 2007). Soybean oil contains approximately $8 \%$ of linolenic acid, LNA (Zobiole et al., 2010), which is an unstable fatty acid that can be easily oxidized, therefore cross-breeding and genetic modification of soybeans have been developed to reduce LNA levels, although LNA (along with linoleic acid (LA)) are essential fatty acids regarding human health, and its amounts in soybean supply the daily LNA intake requirement (Lee et al., 2003). It is also important to remind that LNA is converted into other biologically relevant long chain-polyunsaturated fatty acids ( $n-3$ LC-PUFA) by a sequential desaturation and elongation enzyme system (Almeida et al., 2009).

From other point of view, the presence of monounsaturated fatty acids (MUFA) such as oleic acid in the soy lipidic fraction is highly desirable because it is less prone to oxidation than PUFA. Thus, soybean genotypes with low LNA and high oleic acid quantities are significantly prioritized by food in order to achieve a soybean oil with greater natural oxidative stability (Kumar et al., 2006).

A study in the scientific community reinforced the connection between cardiac health and altered lipids from soybeans, showing that a 
consumption of soy oil with amounts of oleic acid and low percentages of LNA/saturated fatty acids (SFA) instead of the same oil in a mainly hydrogenated state resulted in an improved plasma lipid profile (Lichtenstein et al., 2006). In contrast, as a result of the deleterious effects for human health which are generated by combined high intakes of omega 6 (n-6) fatty acids with relative low consumption of omega 3 (n-3 PUFA) by most people worldwide, medical councils in several countries have recommended a greater daily intake of n-3 PUFA-enriched foods (Carbonera et al., 2014; Tanamati et al., 2009). In this aspect, the transgenic technique can contribute expressively to the genetic improvement of plants for the production of foods with better nutritional quality (Nodari \& Guerra, 2003). However, the influence of planting location on such quality must be studied along with the enhancements from transgenic methods.

Therefore, this study aimed to evaluate a total of 20 new soybean cultivars, non-transgenic and transgenic, destined for human and animal feeding. These studies were conducted in order to characterize the lipid composition of different cultivars and the effect of two different sowing locations on oil content and fatty acid composition. The different cultivars were developed by the Brazilian Agricultural Research Corporation (EMBRAPA Soja). Studies on these genotypes are necessary to characterize this raw material for different utilizations.

\section{Materials and methods}

\section{Sampling}

A total of 20 soybean (G. $\max$ (L.) Merrill) new genotypes, 14 nontransgenic cultivars and 6 transgenic cultivars (which are more resistant to the effects of plagues/diseases), were cultivated by Brazilian Agricultural Research Corporation (EMBRAPA). The experiment was carried out in 2 experimental farms: I (Londrina's region, $23^{\circ} 11^{\prime} \mathrm{S}, 51^{\circ} 11 \mathrm{~W}$, average temperature of $22.8^{\circ} \mathrm{C}, 630 \mathrm{~m}$ of altitude) and II - Ponta Grossa's region, $25^{\circ}, 09^{\prime} \mathrm{S}, 50^{\circ} 04^{\prime} \mathrm{W}$, average temperature of $20,9^{\circ} \mathrm{C}, 884 \mathrm{~m}$ of altitude, both on the Parana state, Brazil, located in southern of Brazil. These regions were chosen because both are featured as great soybean producers and exporters. In such locations, fertile soil was corrected to $\mathrm{pH}$ 5.0. $\mathrm{Al}=0.0, \mathrm{~K}=0.43, \mathrm{Ca}=2.97, \mathrm{Mg}=1.29$, and $\mathrm{H}+\mathrm{Al}=$ $10.45 \mathrm{cmolc} / \mathrm{dm}^{3}, \mathrm{C}=22.75 \mathrm{~g} / \mathrm{dm}^{3}$, and $\mathrm{P}=11.96 \mathrm{mg} / \mathrm{dm}^{3}$ were analyzed.

The soybean varieties under study were grown in soil amended with lime and irrigation if necessary. For every cultivar, two different batches with each one representing an annual harvest (2008 and 2009) were chosen. Each batch was composed of soybean seeds which were randomly collected from the field. Sample handling conditions were identical at the two experimental farms. Soybean seed samples $(0.5 \mathrm{~kg})$ were dried for 5 days at $50{ }^{\circ} \mathrm{C}$ in a ventilated oven and then ground in a coffee grinder. Oil was extracted from ground soybean samples using the Bligh and Dyer (1959). After extraction, the oil was transferred to 1 dram amber vials covered with Argon gas and placed in a freezer $\left(-18{ }^{\circ} \mathrm{C}\right)$ and were mixed. Then, after mixing, a representative amount was selected for posterior analysis. The parameters during soybean development are showed in Table 1.

\section{Fatty acids analysis}

Fatty acid methyl esters (FAME) were prepared by methylation of total lipids by ISO (1978). FAME were analyzed in a Varian model CP-3380 by gas chromatography equipped with a flame ionization detector and fitted a fused silica capillary column CP-720 (100 $\mathrm{m} \times 0.25 \mathrm{~mm}$ i.d. $\times 0.25 \mu \mathrm{m}, 100 \%$ bonded cyanopropyl - Varian, EUA). The gas flow rates (White Martins) used were $1.4 \mathrm{~mL} \mathrm{~min}^{-1}$ carrier

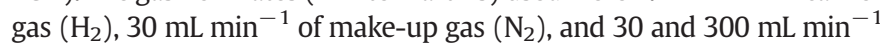
flame gases, $\mathrm{H}_{2}$ and flame synthetic air, respectively. The injector and detector temperatures were at $235^{\circ} \mathrm{C}$. The column temperature was programmed to $65{ }^{\circ} \mathrm{C}$ for $4 \mathrm{~min}$, followed by a ramp of $16{ }^{\circ} \mathrm{C} \mathrm{min}-1$ up to $185^{\circ} \mathrm{C}$, which was kept for $12 \mathrm{~min}$. A second ramp of $20^{\circ} \mathrm{C} \mathrm{min}^{-1}$ was run up to $235^{\circ} \mathrm{C}$ for $14 \mathrm{~min}$. The total analysis time was $40 \mathrm{~min}$. The peak areas were determined using Software Star 5.0 (Varian). The split injection mode used was $1 / 100$ and injections of $1 \mu \mathrm{L}$ were performed in triplicate. FAME identification was made by comparison with the retention times of standards from Sigma (St. Louis, MO, USA) and equivalent chain-length values and fatty acid composition were expressed as weight percentage of each fatty acid to the total fatty acids. The analyses were conducted in three replicates.

\section{Statistical analysis}

Statistical analysis was performed using Statistica 7.0 software, and refers to the average of three tests. Comparisons were done with respect to their similarities by Tukey's test for samples of each region and also for different regions. Means with the same letter in each row are not significantly different $(P>0.05)$. The averages with equal numbers have no significant differences by region of planting $(P>0.05)$. Principal component analysis (PCA) analysis was performed using Statistica 7.0 software and the pre-treatment of data was not necessary.

\section{Results and discussion}

Tables 2, 3, 4 and 5 show the 11 fatty acids which were detected in non-transgenic and transgenic samples of soybean produced in different regions. They are: 14:0, 16:0, 16:1n-9, 17:0, 18:0, 18:1n-7, 18:1n-9, 18:2n-6, 18:3n-3, 20:0 and 24:0. The same fatty acid types were observed in every studied bean, however, with significant variation of their amounts in different samples from different studied regions.

The highest PUFA amounts were achieved for the transgenic and non-transgenic beans produced in the Londrina's and Ponta Grossa's region, respectively. However, results of monounsaturated and saturated

Table 1

Locations, cultivars, and weather conditions during soybean development in the cropping season 2008/2009.

\begin{tabular}{|c|c|c|c|}
\hline Location & Cultivars & Rainfall (mm) & Average Temperature $\left({ }^{\circ} \mathrm{C}\right)$ \\
\hline $\begin{array}{l}\text { Londrina's region } \\
23^{\circ} 11^{\prime} 37^{\prime \prime} \text { (latitude) } \\
51^{\circ} 11^{\prime} 03^{\prime \prime} \text { (longitude) } \\
630 \text { meters (altitude) }\end{array}$ & 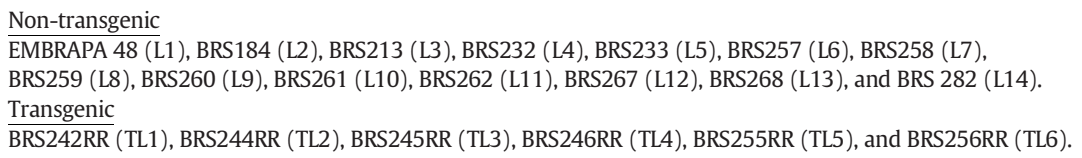 & 726.6 & 22.8 \\
\hline $\begin{array}{l}\text { Ponta Grossa's region } \\
25^{\circ} 09^{\prime} 31^{\prime \prime} \text { (latitude) } \\
50^{\circ} 04^{\prime} 32^{\prime \prime} \text { (longitude) } \\
884 \text { meters (altitude) }\end{array}$ & 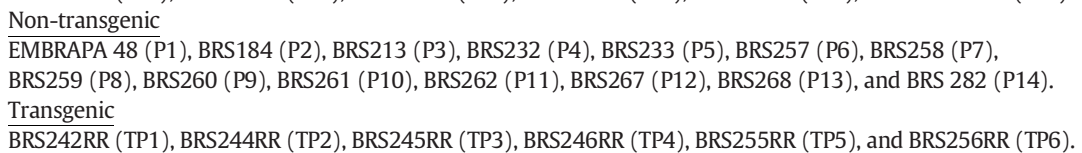 & 588.8 & 20.9 \\
\hline
\end{tabular}

$\mathrm{L}=$ Londrina; $\mathrm{P}=$ Ponta Grossa; $\mathrm{TL}=$ transgenic Londrina's region; $\mathrm{TP}=$ transgenic Ponta Grossa's region. 
Table 2

Fatty acids composition (mg/g of total lipids) of non-transgenic soybean produced in the Londrina's region, Parana State, Brazil ${ }^{1}$.

\begin{tabular}{|c|c|c|c|c|c|c|c|c|c|c|c|c|c|c|c|}
\hline Fatty acids & Embrapa 48 & BRS 184 & BRS 213 & BRS 232 & BRS 233 & BRS 257 & BRS 258 & BRS 259 & BRS 260 & BRS 261 & BRS 262 & BRS 267 & BRS 268 & BRS 282 & SD Max \\
\hline 14:0 & $0.07^{\mathrm{f1}}$ & $0.23^{b}$ & $0.13^{\mathrm{d}}$ & $0.26^{\mathrm{ab}}$ & $0.13^{\mathrm{d}}$ & $0.20^{c}$ & $0.25^{\mathrm{ab}}$ & $0.11^{\mathrm{de}}$ & $0.25^{\mathrm{ab}}$ & $0.28^{\mathrm{a}}$ & $0.09^{\mathrm{ef}}$ & $0.09^{\mathrm{ef}}$ & $0.08^{\mathrm{f}}$ & $0.09^{e f}$ & 0.02 \\
\hline 16:0 & $12.62^{e f}$ & $12.56^{\mathrm{f}}$ & $13.44^{\mathrm{a}}$ & $13.48^{\mathrm{a}}$ & $12.69^{\mathrm{e}}$ & $13.34^{\mathrm{b}}$ & $13.05^{\mathrm{c}}$ & $13.02^{\mathrm{c}}$ & $12.90^{\mathrm{d}}$ & $12.93^{\mathrm{d}}$ & $11.42^{\mathrm{kl}}$ & $11.07^{\mathrm{m}}$ & $10.06^{\mathrm{n}}$ & $11.51^{\mathrm{k}}$ & 0.43 \\
\hline 16:1n-9 & $0.08^{\mathrm{cd} 1}$ & $0.07^{\mathrm{de}}$ & $0.06^{\mathrm{e}}$ & $0.06^{\mathrm{e}}$ & $0.06^{\mathrm{e} 2}$ & $0.06^{\mathrm{e} 3}$ & $0.07^{\text {de } 4}$ & $0.07^{\text {de } 5}$ & $0.07^{\text {de } 6}$ & $0.06^{\mathrm{e}}$ & $0.07^{\mathrm{e} 8}$ & $0.06^{e 9}$ & $0.10^{\mathrm{ab} 10}$ & $0.07^{\mathrm{de} 11}$ & 0.02 \\
\hline 17:0 & $0.13^{\mathrm{b}}$ & $0.09^{\text {cd }}$ & $0.10^{\mathrm{c} 1}$ & $0.15^{\mathrm{a}}$ & $0.01^{\mathrm{e}}$ & $0.01^{\mathrm{e}}$ & $0.09^{\mathrm{cd} 2}$ & $0.10^{c 3}$ & $0.09^{\mathrm{cd} 4}$ & $0.09^{\mathrm{cd} 4}$ & $0.08^{\mathrm{d} 5}$ & $0.09^{\text {cd } 6}$ & $0.10^{c}$ & $0.09^{\text {cd7 }}$ & 0.02 \\
\hline 18:0 & $3.61^{\mathrm{ef}}$ & $4.06^{\mathrm{ab}}$ & $3.74^{\text {de } 1}$ & $3.59^{\mathrm{efg}}$ & $4.05^{\mathrm{ab}}$ & $4.03^{\mathrm{ab} 2}$ & $3.60^{\mathrm{ef} 3}$ & $3.46^{\text {fghi }}$ & $3.75^{\text {cde }}$ & $3.93^{\mathrm{bc}}$ & $3.42^{\text {ghi4 }}$ & $3.19^{\mathrm{j}}$ & $3.84^{\mathrm{cd} 5}$ & $3.37^{\text {hij }}$ & 0.10 \\
\hline 18:1n-9 & $16.71^{\mathrm{bc}}$ & $17.94^{\mathrm{a}}$ & $15.07^{\mathrm{efg}}$ & $15.99^{\text {cde }}$ & $13.98^{\mathrm{fgh}}$ & $14.48^{\text {fgh1 }}$ & $15.10^{\mathrm{efg}}$ & $15.62^{\text {cdef2 }}$ & $15.09^{\operatorname{efg} 3}$ & $15.50^{\operatorname{def} 4}$ & $16.46^{\mathrm{bcd} 5}$ & $13.46^{\mathrm{hij}}$ & $18.06^{\mathrm{a}}$ & $14.46^{\text {fgh }}$ & 0.76 \\
\hline 18:1n-7 & $1.32^{\mathrm{ef} 1}$ & $1.43^{\mathrm{e} 2}$ & $1.31^{\mathrm{ef3}}$ & $1.23^{\mathrm{f} 4}$ & $1.21^{\mathrm{f}}$ & $1.25^{\mathrm{f5}}$ & $1.26^{\mathrm{f6}}$ & $1.73^{\text {def7}}$ & $1.26^{\mathrm{f8}}$ & $1.34^{\mathrm{ef}}$ & $2.82^{\mathrm{abc}}$ & $3.40^{\mathrm{a}}$ & $2.49^{\mathrm{bcd}}$ & $2.86^{\mathrm{abc}}$ & 0.54 \\
\hline $18: 2 n-6$ & $57.32^{\text {abcde } 1}$ & $55.87^{\mathrm{bcde} 2}$ & $57.86^{\text {abcd3 }}$ & $55.83^{\text {bcde } 4}$ & $59.23^{\mathrm{a} 5}$ & $58.60^{\mathrm{abcd} 6}$ & $59.02^{\mathrm{ab}}$ & $57.33^{\text {abcde } 7}$ & $58.89^{\mathrm{abc}}$ & $57.58^{\text {abcde }}$ & $56.65^{\text {abcde9 }}$ & $56.19^{\text {abcde } 10}$ & $55.47^{\mathrm{de} 11}$ & $58.79^{\mathrm{abc} 12}$ & 2.09 \\
\hline $18: 3 n-3$ & $7.99^{\text {def1 }}$ & $7.56^{\mathrm{f} 2}$ & $8.11^{\text {cdef3 }}$ & $9.13^{\text {bcdef } 4}$ & $8.47^{\text {bcdef5 }}$ & $7.86^{\text {def6 }}$ & $7.38^{\mathrm{f}}$ & $8.42^{\text {cdef7 }}$ & $7.52^{\mathrm{fB}}$ & $8.10^{\text {def9 }}$ & $8.43^{\text {cdef } 10}$ & $11.89^{\mathrm{a}}$ & $9.27^{\text {bcdef } 11}$ & $8.28^{\text {cdef } 12}$ & 1.43 \\
\hline 20:0 & $0.01^{\mathrm{d} 1}$ & $0.01^{\mathrm{d} 2}$ & $0.01^{\mathrm{d} 3}$ & $0.10^{c}$ & $0.01^{\mathrm{d} 4}$ & $0.01^{\mathrm{d} 5}$ & $0.02^{\mathrm{d} 6}$ & $0.01^{\mathrm{d}}$ & $0.01^{\mathrm{d} 7}$ & $0.01^{\mathrm{d} 8}$ & $0.23^{\mathrm{a}}$ & $0.17^{\mathrm{b}}$ & $0.17^{\mathrm{b}}$ & $0.18^{\mathrm{b}}$ & 0.09 \\
\hline 24:0 & $0.15^{\mathrm{de} 1}$ & $0.17^{\text {bcde2 }}$ & $0.17^{\text {bcde3 }}$ & $0.16^{\text {cde } 4}$ & $0.16^{\text {cde5 }}$ & $0.16^{\text {cdef6 }}$ & $0.16^{\text {cde } 7}$ & $0.12^{e 8}$ & $0.16^{\text {cde9 }}$ & $0.16^{\text {cde10 }}$ & $0.31^{\text {abcde1 }}$ & $0.41^{\mathrm{a}}$ & $0.37^{\mathrm{abcd}}$ & $0.29^{\text {abcde } 12}$ & 0.03 \\
\hline SFA & $16.58^{\text {ef }}$ & $17.13^{\mathrm{f}}$ & $17.60^{\mathrm{a}}$ & $17.75^{\mathrm{a}}$ & $17.05^{\mathrm{e}}$ & $17.75^{\mathrm{b}}$ & $17.18^{\mathrm{c}}$ & $16.83^{\mathrm{c}}$ & $17.16^{\mathrm{d}}$ & $17.41^{\mathrm{d}}$ & $15.56^{\mathrm{kl}}$ & $15.01^{\mathrm{m}}$ & $14.61^{\mathrm{n}}$ & $15.54^{\mathrm{k}}$ & 0.43 \\
\hline MUFA & $18.11^{\mathrm{bc}}$ & $19.44^{\mathrm{a}}$ & $16.43^{\text {efg }}$ & $17.29^{\text {cde }}$ & $15.25 \mathrm{fgh}$ & $15.78^{\text {fgh1 }}$ & $16.43^{\mathrm{efg}}$ & $17.42^{\text {cdef2 }}$ & $16.42^{\text {efg3 }}$ & $16.90^{\operatorname{def} 4}$ & $19.36^{\text {bcd5 }}$ & $16.92^{\mathrm{hij}}$ & $20.65^{\mathrm{a}}$ & $17.39^{\mathrm{fgh}}$ & 0.76 \\
\hline PUFA & $65.31^{\text {de } 1}$ & $63.43^{\mathrm{ef} 2}$ & $65.97^{\mathrm{cd} 3}$ & $64.96^{\text {bcde } 4}$ & $67.70^{\mathrm{Ab5}}$ & $66.4^{\mathrm{d} 6}$ & $66.40^{\text {af }}$ & $65.75^{\text {cde } 7}$ & $66.41^{\mathrm{cf8}}$ & $65.68^{\text {de9 }}$ & $65.08^{\text {cde9 }}$ & $68.08^{\mathrm{a} 10}$ & $64.74^{\mathrm{de} 11}$ & $67.07^{112}$ & 2.53 \\
\hline$n-6 / n-3$ & $7.17^{\mathrm{cd}}$ & $7.39^{\mathrm{bc}}$ & $7.14^{\mathrm{cd} 1}$ & $6.12^{\mathrm{f2}}$ & $6.99^{\mathrm{de}}$ & $7.46^{\mathrm{b}}$ & $8.00^{\mathrm{a}}$ & $6.81^{\mathrm{e}}$ & $7.83^{\mathrm{a}}$ & $7.11^{\mathrm{cd} 3}$ & $6.72^{\mathrm{e}}$ & $4.73^{\mathrm{j}}$ & $5.99^{\mathrm{fg}}$ & $7.10^{\text {de } 4}$ & 0.16 \\
\hline
\end{tabular}

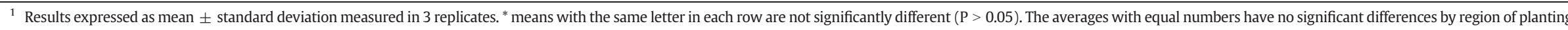
$(\mathrm{P}>0.05)$.

Table 3

Fatty acids composition (mg/g of total lipids) of non-transgenic soybean produced in the Ponta Grossa's region, Parana State, Brazil ${ }^{1}$.

\begin{tabular}{|c|c|c|c|c|c|c|c|c|c|c|c|c|c|c|c|}
\hline Fatty acids & EMBRAPA 48 & BRS 184 & BRS 213 & BRS 232 & BRS 233 & BRS 257 & BRS 258 & BRS 259 & BRS 260 & BRS 261 & BRS 262 & BRS 267 & BRS 268 & BRS 282 & SD Max \\
\hline 14:0 & $0.05^{\mathrm{hij} 1}$ & $0.06^{\text {cdefg }}$ & $0.07^{\mathrm{b}}$ & $0.10^{\mathrm{a}}$ & $0.05^{\text {ghi }}$ & $0.07^{\mathrm{bcd}}$ & $0.06^{\text {cdefg }}$ & $0.06^{\text {bcde }}$ & $0.05^{\mathrm{hij}}$ & $0.05^{\mathrm{ij}}$ & $0.06^{\text {defg }}$ & $0.05^{\text {fghi }}$ & $0.04^{\mathrm{j}}$ & $0.06^{\text {defg }}$ & 0.01 \\
\hline 16:0 & $11.75^{\mathrm{i}}$ & $11.90^{\mathrm{h}}$ & $12.42^{\mathrm{e}}$ & $11.97^{\text {gh }}$ & $13.04^{\mathrm{b}}$ & $12.74^{\mathrm{c}}$ & $12.74^{\mathrm{c}}$ & $13.28^{\mathrm{a}}$ & $12.61^{\mathrm{d}}$ & $11.24^{1}$ & $11.66^{\mathrm{ij}}$ & $12.00^{\mathrm{g}}$ & $10.86^{\mathrm{m}}$ & $11.93^{\text {gh }}$ & 0.35 \\
\hline $16: 1 n-9$ & $0.08^{\mathrm{bc} 1}$ & $0.01^{\mathrm{d}}$ & $0.01^{\mathrm{e}}$ & $0.08^{\mathrm{bc}}$ & $0.07^{\mathrm{cd} 2}$ & $0.06^{\mathrm{d} 3}$ & $0.08^{\mathrm{bc} 4}$ & $0.08^{\mathrm{bc5}}$ & $0.08^{\mathrm{bc} 6}$ & $0.07^{\mathrm{cd} 7}$ & $0.08^{\mathrm{bc} 8}$ & $0.07^{\mathrm{cd} 9}$ & $0.09^{\mathrm{ab} 10}$ & $0.07^{\mathrm{cd} 11}$ & 0.01 \\
\hline 17:0 & $0.10^{\text {cd }}$ & $0.11^{\mathrm{bc}}$ & $0.10^{\mathrm{d} 1}$ & $0.09^{\mathrm{d}}$ & $0.10^{\text {cd }}$ & $0.10^{\text {cd }}$ & $0.09^{\mathrm{d} 2}$ & $0.09^{\mathrm{d} 3}$ & $0.09^{\mathrm{d} 4}$ & $0.11^{\mathrm{bc} 7}$ & $0.09^{\mathrm{d} 5}$ & $0.10^{\text {cd } 6}$ & $0.15^{\mathrm{a}}$ & $0.10^{\mathrm{cd} 7}$ & 0.01 \\
\hline 18:0 & $3.96^{\mathrm{ef}}$ & $4.62^{\mathrm{a}}$ & $3.61^{\mathrm{j} 1}$ & $3.43^{1}$ & $3.89^{\mathrm{fg}}$ & $4.03^{\mathrm{e}^{2}}$ & $3.66^{\mathrm{ij} 3}$ & $3.82^{\mathrm{gh}}$ & $3.33^{\mathrm{m}}$ & $3.65^{\mathrm{ij}}$ & $3.46^{14}$ & $3.7^{4 h i}$ & $3.79^{\mathrm{h5}}$ & $3.62^{\mathrm{j}}$ & 0.05 \\
\hline 18:1n-9 & $18.30^{\mathrm{cd}}$ & $20.63^{\mathrm{b}}$ & $17.04^{\text {defg }}$ & $19.38^{\mathrm{bc}}$ & $15.56^{\text {ghi }}$ & $15.08^{\text {hi1 }}$ & $17.60^{\mathrm{def}}$ & $16.22^{\text {fgh2 }}$ & $14.35^{\mathrm{i}}$ & $16.79^{\text {efg } 4}$ & $17.07^{\text {def5 }}$ & $18.45^{\mathrm{cd}}$ & $20.48^{\mathrm{b}}$ & $17.71^{\mathrm{def}}$ & 1.03 \\
\hline 18:1n-7 & $1.59^{\text {cde } 1}$ & $1.33^{\mathrm{g} 2}$ & $1.58^{\text {cdef3 }}$ & $1.50^{\operatorname{defg} 4}$ & $1.91^{\mathrm{b}}$ & $1.70^{\text {bcdes }}$ & $1.74^{\mathrm{bcd} 6}$ & $1.70^{\text {bcde } 7}$ & $1.92^{\mathrm{b} 8}$ & $2.42^{\mathrm{a}}$ & $1.48^{\mathrm{efg}}$ & $1.60^{\text {cde }}$ & $1.77^{\mathrm{bc}}$ & $1.33^{\mathrm{g}}$ & 0.19 \\
\hline $18: 2 n-6$ & $55.45^{\text {bcdef1 }}$ & $53.32^{\operatorname{defg} 2}$ & $56.91^{\mathrm{abc} 3}$ & $54.43^{\text {cdefg } 4}$ & $56.17^{\text {abd5 }}$ & $57.82^{\mathrm{ab} 6}$ & $54.61^{\text {cdefg }}$ & $55.20^{\text {bcdef } 7}$ & $59.34^{\mathrm{a}}$ & $57.40^{\mathrm{abc} 8}$ & $56.46^{\text {abcd9 }}$ & $55.62^{\text {bcde } 10}$ & $55.12^{\text {bcdef11 }}$ & $57.01^{\mathrm{abc} 12}$ & 0.99 \\
\hline $18: 3 n-3$ & $8.56^{\text {bcde1 }}$ & $7.88^{\mathrm{e} 2}$ & $8.11^{\text {def3 }}$ & $8.87^{\mathrm{abcd} 4}$ & $9.08^{\mathrm{abc} 5}$ & $8.25^{\text {cdeff }}$ & $9.28^{\mathrm{ab}}$ & $9.20^{\mathrm{ab} 7}$ & $8.10^{\text {def8 }}$ & $8.12^{\text {def9 }}$ & $9.51^{\mathrm{a} 10}$ & $8.22^{\text {cdef }}$ & $7.56^{f 11}$ & $8.01^{\text {def12 }}$ & 0.17 \\
\hline $20: 0$ & $0.03^{c^{1}}$ & $0.01^{\mathrm{f} 2}$ & $0.01^{\mathrm{ef} 3}$ & $0.01^{\mathrm{f}}$ & $0.02^{\mathrm{d} 4}$ & $0.03^{c 5}$ & $0.01^{\mathrm{f} 6}$ & $0.22^{\mathrm{a}}$ & $0.02^{\mathrm{de} 7}$ & $0.02^{\mathrm{de} 8}$ & $0.00^{\mathrm{g}}$ & $0.01^{\mathrm{f}}$ & $0.01^{\mathrm{ef}}$ & $0.01^{\mathrm{f}}$ & 0.00 \\
\hline 24:0 & $0.14^{\mathrm{ab} 1}$ & $0.13^{\mathrm{bc} 2}$ & $0.14^{\mathrm{ab} 3}$ & $0.15^{\mathrm{a} 4}$ & $0.12^{c 5}$ & $0.13^{\mathrm{bc} 6}$ & $0.13^{\mathrm{bc} 7}$ & $0.12^{c 8}$ & $0.12^{\mathrm{c} 9}$ & $0.13^{\mathrm{bc} 10}$ & $0.14^{\mathrm{ab} 11}$ & $0.14^{\mathrm{ab}}$ & $0.13^{\mathrm{bc}}$ & $0.14 \mathrm{ab} 12$ & 0.00 \\
\hline SFA & $16.03^{\mathrm{i}}$ & $16.83^{\text {h }}$ & $16.34^{\mathrm{e}}$ & $15.75^{\text {gh }}$ & $17.21^{\mathrm{b}}$ & $17.10^{c}$ & $16.70^{\mathrm{a}}$ & $17.60^{\mathrm{d}}$ & $16.22^{1}$ & $15.20^{1}$ & $15.41^{\mathrm{ij}}$ & $16.04^{\mathrm{g}}$ & $14.98^{\mathrm{m}}$ & $15.87^{\text {gh }}$ & 0.35 \\
\hline MUFA & $19.97^{\mathrm{cd}}$ & $21.97^{\mathrm{b}}$ & $18.64^{\mathrm{defg}}$ & $20.96^{\mathrm{bc}}$ & $17.54^{\text {ghi }}$ & $16.83^{\text {hi1 } 1}$ & $19.41^{\text {def }}$ & $18.00^{\mathrm{fgh} 2}$ & $16.34^{\text {efg } 4}$ & $19.27^{\text {efg4 }}$ & $18.62^{\mathrm{def5}}$ & $20.12^{\mathrm{cd}}$ & $22.34^{\mathrm{b}}$ & $19.11^{\mathrm{def}}$ & 1.03 \\
\hline PUFA & $64.01^{\mathrm{bcd} 1}$ & $61.20^{\mathrm{ef} 2}$ & $65.02^{\mathrm{cd} 3}$ & $63.30^{\mathrm{cd} 4}$ & $65.25^{c 5}$ & $66.07^{\mathrm{bc} 6}$ & $63.89^{\mathrm{ac}}$ & $64.40^{\mathrm{b} 7}$ & $67.44^{\mathrm{ad} 8}$ & $65.52^{\mathrm{cd} 8}$ & $65.97^{\mathrm{a} 9}$ & $63.84^{\mathrm{de} 10}$ & $62.68^{f 11}$ & $65.02^{\mathrm{cd} 12}$ & 2.13 \\
\hline$n-6 / n-3$ & $6.48^{\mathrm{d}}$ & $6.77^{\mathrm{c}}$ & $7.02^{\mathrm{b} 1}$ & $6.14^{\mathrm{ef2}}$ & $6.19^{\mathrm{e}}$ & $7.01^{\mathrm{b}}$ & $5.89^{\mathrm{h}}$ & $6.00^{\text {gh }}$ & $7.32^{\mathrm{a}}$ & $7.07^{\mathrm{b3}}$ & $5.94^{\text {gh }}$ & $6.77^{c}$ & $7.29^{\mathrm{a}}$ & $7.11^{\mathrm{b} 4}$ & 0.03 \\
\hline
\end{tabular}

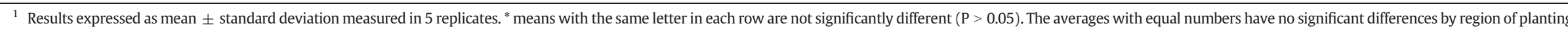
$(\mathrm{P}>0.05)$. 
Table 4

Fatty acids composition (mg/g of total lipids) of transgenic soybean produced in the Londrina's region, Parana State, Brazil ${ }^{1}$.

\begin{tabular}{|c|c|c|c|c|c|c|c|}
\hline Fatty acids & BRS 242RR & BRS 244RR & BRS 245RR & BRS 246RR & BRS 255RR & BRS 256RR & SD Max \\
\hline 14:0 & $0.09^{\mathrm{ef}}$ & $0.10^{\text {ef }}$ & $0.09^{\mathrm{ef}}$ & $0.10^{\mathrm{ef}}$ & $0.09^{\mathrm{ef}}$ & $0.09^{\mathrm{ef}}$ & 0.01 \\
\hline $16: 0$ & $12.13^{\mathrm{h}}$ & $12.38^{\mathrm{g}}$ & $11.92^{\mathrm{i}}$ & $11.94^{\mathrm{i}}$ & $11.64^{\mathrm{j}}$ & $11.34^{1}$ & 0.39 \\
\hline $16: 1 n-9$ & $0.09^{\mathrm{bc} 12}$ & $0.07^{\mathrm{de} 13}$ & $0.11^{\mathrm{a}}$ & $0.08^{\mathrm{cd}}$ & $0.09^{\text {bc14 }}$ & $0.08^{\mathrm{cd}}$ & 0.02 \\
\hline $17: 0$ & $0.09^{\mathrm{cd} 8}$ & $0.10^{c}$ & $0.10^{\mathrm{c} 9}$ & $0.09^{\operatorname{cd} 10}$ & $0.09^{\mathrm{cd} 11}$ & $0.09^{\mathrm{cd} 12}$ & 0.02 \\
\hline 18:0 & $4.10^{\mathrm{ab}}$ & $4.19^{\mathrm{a}}$ & $3.75^{\text {cde }}$ & $3.53^{\text {fgh } 6}$ & $3.30^{\mathrm{ij}}$ & $3.53^{\mathrm{fgh}}$ & 0.07 \\
\hline $18: 1 n-9$ & $12.33^{\mathrm{jk}}$ & $12.14^{\mathrm{k}}$ & $12.98^{\mathrm{ijk}}$ & $14.81^{\text {efg }}$ & $17.92^{\mathrm{a}}$ & $17.51^{\mathrm{ab} 6}$ & 0.86 \\
\hline $18: 1 n-7$ & $2.90^{\mathrm{abc}}$ & $2.46^{\text {bcd }}$ & $2.16^{\text {cde9 }}$ & $2.55^{\mathrm{abcd}}$ & $3.11^{\mathrm{ab}}$ & $2.40^{\mathrm{bcd}}$ & 0.60 \\
\hline $18: 2 n-6$ & $56.01^{\text {abcde } 13}$ & $57.53^{\text {abcde } 14}$ & $57.97^{\text {abcd9 }}$ & $56.31^{\text {abcde }}$ & $55.67^{\text {cde }}$ & $54.46^{\mathrm{e} 15}$ & 2.58 \\
\hline $18: 3 n-3$ & $11.82^{\mathrm{a}}$ & $10.71^{\mathrm{ab} 13}$ & $10.37^{\mathrm{abc}}$ & $10.04^{\mathrm{abcd}}$ & $7.77^{\mathrm{ef} 14}$ & $9.95^{\text {abcde } 15}$ & 1.81 \\
\hline 20:0 & $0.16^{\mathrm{b}}$ & $0.16^{\mathrm{b}}$ & $0.18^{\mathrm{b}}$ & $0.18^{\mathrm{b}}$ & $0.18^{\mathrm{b}}$ & $0.18^{\mathrm{b}}$ & 0.01 \\
\hline 24:0 & $0.27^{\text {abcde } 13}$ & $0.17^{\text {bcde } 14}$ & $0.3^{\mathrm{abc}}$ & $0.39^{\mathrm{ab}}$ & $0.14^{\mathrm{e} 15}$ & $0.37^{\text {abcd }}$ & 0.14 \\
\hline SFA & $16.85^{\mathrm{h}}$ & $17.10^{\mathrm{g}}$ & $16.41^{\mathrm{i}}$ & $16.22^{\mathrm{i}}$ & $15.44^{\mathrm{j}}$ & $15.60^{1}$ & 0.43 \\
\hline MUFA & $15.32^{\mathrm{jk}}$ & $14.67^{\mathrm{k}}$ & $15.24^{\mathrm{ijk}}$ & $17.43^{\text {efg }}$ & $21.12^{\mathrm{a}}$ & $19.99^{\mathrm{ab} 6}$ & 0.76 \\
\hline PUFA & $67.83^{\mathrm{a} 13}$ & $68.24^{\mathrm{ab} 14}$ & $68.34^{\mathrm{c} 9}$ & $66.35^{\mathrm{abcd}}$ & $63.44^{\mathrm{e} 14}$ & $64.41^{\mathrm{e} 15}$ & 3.15 \\
\hline$n-6 / n-3$ & $4.74^{\mathrm{j}}$ & $5.37^{\mathrm{i}}$ & $5.59^{\mathrm{gh}}$ & $5.61^{\mathrm{hi}}$ & $7.17^{\mathrm{cd}}$ & $5.47^{\mathrm{i}}$ & 0.17 \\
\hline
\end{tabular}

1 Results expressed as mean \pm standard deviation measured in 5 replicates. *Varieties codified as RR (Roundup resistant) are transgenic soybeans. BRS 242 RR - EMBRAPA 58 * 5 X (E96-

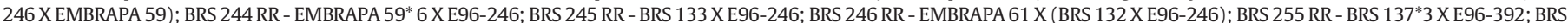

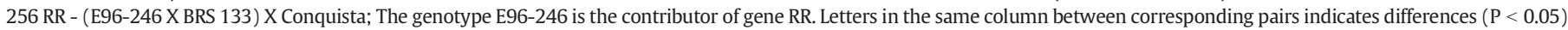
by Tukey's test.

fatty acids varied between in non-transgenic and transgenic samples produced in different regions. Recent studies with transgenic and nontransgenic soybean developed by Milinski et al. (2007) showed similar results.

The predominant SFA in both conventional and transgenic soybean varieties were palmitic (16:0) and stearic (18:0) acids while the most significant monounsaturated fatty acids were oleic (18:1n-9) and cisvaccenic (18:1n-7) acids. According to Daniels, Kim, and Min (2006), the linoleic acid level in soy oil also was higher than other fatty acids found in their recent studies. The same trend was observed in this article.

The non-transgenic varieties BRS 184, BRS 232, BRS 257, BRS 258, BRS 260 and BRS 261 grown in Londrina region showed the highest quantities of 14:0 fatty acid. Nevertheless, in the BRS 213/BRS 232/BRS 237 varieties from Londrina region and in the BRS 233/BRS 259 varieties cultivated in Ponta Grossa region, they showed higher results of palmitic acid (16:0), when compared with other varieties and regions. The highest concentration of oleic acid was found (about $20 \mathrm{mg} \mathrm{g}^{-1}$ of lipids) in the BRS 184 and BRS 268 varieties from Ponta Grossa region.
Non-transgenic samples from Londrina region had a higher average content of 16:0 than those of Ponta Grossa's, while 16:0 means of transgenic varieties showed an opposite trend. Transgenic varieties from Londrina's region showed higher contents of $18: 1 \mathrm{n}-7$ in relation to Ponta Grossa's, this superiority was also confirmed for 18:1n-9, 18:2n-6, 18:3n-3, 20:0 and 24:0 fatty acids. For samples of Londrina's and Ponta Grossa's regions, total unsaturated fatty acid percentages were higher than $82 \%$. In relation to n-3 fatty acids, there was an increase in transgenic species, and consequently, lower $n-6 / n-3$ ratios were obtained, which is highly advantageous for the consumers' health. The importance of ingesting products that are rich in PUFA n-3 and also the beneficial effects of a high ratio n-3 to n- 6 has been reported in the literature (Bonafe et al., 2011).

Multivariate analysis can summarize the variability of a complex data set and present it in a most interpretable form, such as principal components (PC) (Ribeiro et al., 2013; Wu, Rodgers, \& Marshall, 2004). The PC analysis of the soybean samples was carried out because vegetable oils are very complex mixtures (Wu et al., 2004). This two-dimensional representation provides a scattering of samples, according to their relative

Table 5

Fatty acids composition (mg/g of total lipids) of transgenic soybean produced in the Ponta Grossa's region, Parana State, Brazil ${ }^{1}$.

\begin{tabular}{|c|c|c|c|c|c|c|c|}
\hline Fatty acids & BRS 242RR & BRS 244RR & BRS245R & BRS246RR & BRS 255RR & BRS 256RR & SD Max \\
\hline 14:0 & $0.06^{\text {cdef }}$ & $0.07^{\mathrm{bc}}$ & $0.06^{\text {efgh }}$ & $0.05^{\text {fghi }}$ & $0.06 \mathrm{e}^{\mathrm{fgh}}$ & $0.05^{\mathrm{ij}}$ & 0.01 \\
\hline 16:0 & $12.43^{\mathrm{e}}$ & $12.62^{\mathrm{d}}$ & $12.58^{\mathrm{d}}$ & $11.57^{\mathrm{jk}}$ & $11.48^{\mathrm{k}}$ & $12.10^{f}$ & 0.73 \\
\hline $16: 1 n-9$ & $0.09^{\mathrm{ab} 12}$ & $0.08^{\mathrm{bc} 13}$ & $0.01^{\mathrm{e}}$ & $0.01^{\mathrm{e}}$ & $0.10^{\mathrm{a} 14}$ & $0.01^{\mathrm{d}}$ & 0.00 \\
\hline $17: 0$ & $0.09^{\mathrm{d} 8}$ & $0.12^{\mathrm{b}}$ & $0.09^{\mathrm{d} 9}$ & $0.09^{\mathrm{d} 10}$ & $0.09^{\mathrm{d} 11}$ & $0.10^{\operatorname{cd} 12}$ & 0.01 \\
\hline 18:0 & $4.31 d$ & $4.42^{\mathrm{C}}$ & $4.52^{\mathrm{b}}$ & $3.58^{\mathrm{jk} 6}$ & $3.51^{\mathrm{kl}}$ & $3.93^{\mathrm{f}}$ & 0.21 \\
\hline $18: 1 n-9$ & $19.43^{\mathrm{bc}}$ & $16.71^{\mathrm{efg}}$ & $20.58^{\mathrm{b}}$ & $23.36^{\mathrm{a}}$ & $22.92^{\mathrm{a}}$ & $18.02^{\text {cde6 }}$ & 0.53 \\
\hline $18: 1 n-7$ & $1.34^{\mathrm{fg}}$ & $1.50^{\mathrm{defg}}$ & $1.63^{\text {cde9 }}$ & $1.81^{\mathrm{bc}}$ & $1.90^{\mathrm{b}}$ & $1.47^{\mathrm{efg}}$ & 0.11 \\
\hline $18: 2 n-6$ & $52.85^{\text {efg13 }}$ & $55.18^{\text {bcdef14 }}$ & $52.29^{\mathrm{fg}}$ & $51.43^{\mathrm{g}}$ & $51.67^{\mathrm{g}}$ & $55.69^{\text {bcde15 }}$ & 3.20 \\
\hline $18: 3 n-3$ & $9.27^{\mathrm{ab}}$ & $9.17^{\mathrm{ab} 13}$ & $8.10^{\text {def }}$ & $7.94^{\text {ef }}$ & $8.03^{\text {def14 }}$ & $8.51^{\text {bcde } 15}$ & 0.34 \\
\hline 20:0 & $0.01^{\mathrm{f}}$ & $0.01^{\mathrm{f}}$ & $0.01^{\mathrm{f}}$ & $0.01^{\mathrm{b}}$ & $0.09^{\mathrm{b}}$ & $0.01^{\mathrm{f}}$ & 0.01 \\
\hline 24:0 & $0.12^{\mathrm{c} 13}$ & $0.13^{\mathrm{bc} 14}$ & $0.10^{\mathrm{bc}}$ & $0.14^{\mathrm{ab}}$ & $0.15^{\mathrm{a} 15}$ & $0.13^{\mathrm{bc}}$ & 0.01 \\
\hline SFA & $17.02^{\mathrm{e}}$ & $17.37^{d}$ & $17.39^{d}$ & $15.44^{\mathrm{jk}}$ & $15.38^{\mathrm{k}}$ & $16.31^{\mathrm{f}}$ & 0.73 \\
\hline MUFA & $20.87^{\mathrm{bc}}$ & $18.29^{\text {efg }}$ & $22.21^{\mathrm{b}}$ & $25.19^{a}$ & $24.92^{\mathrm{a}}$ & $19.50^{\text {cde } 6}$ & 0.53 \\
\hline PUFA & $62,12^{\mathrm{b} 13}$ & $64,35^{\text {be } 14}$ & $60,39^{\mathrm{f}}$ & $59,37^{\mathrm{fg}}$ & $59,70^{\mathrm{fg} 14}$ & $64,20^{\mathrm{ef} 15}$ & 3.15 \\
\hline$n-6 / n-3$ & $5,70^{\mathrm{i}}$ & $6,02^{\mathrm{fg}}$ & $6,46^{\mathrm{d}}$ & $6,48^{\mathrm{d}}$ & $6,44^{\mathrm{d}}$ & $6,55^{\mathrm{d}}$ & 0.10 \\
\hline
\end{tabular}

1 Results expressed as mean \pm standard deviation measured in 5 replicates. *Varieties codified as RR (Roundup resistant) are transgenic soybeans. BRS 242 RR - EMBRAPA $58 * 5$ X (E96-

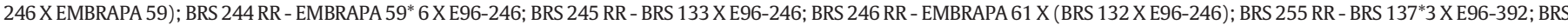

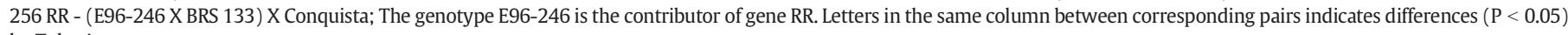
by Tukey's test. 
Table 6

Results of principal component analysis of the non-transgenic and transgenic soybean produced in Londrina's and Ponta Grossa's regions.

\begin{tabular}{|c|c|c|c|c|c|c|}
\hline \multirow[t]{3}{*}{ Value number } & \multicolumn{6}{|c|}{ Londrina's and Ponta Grossa's regions } \\
\hline & \multicolumn{3}{|c|}{ Non-transgenic soybean } & \multicolumn{3}{|c|}{ Transgenic soybean } \\
\hline & Eigenvalues & $\%$ total variance & Cumulative \% & Eigenvalues & $\%$ total variance & Cumulative \% \\
\hline PC1 & 2,41 & 60,24 & 60,24 & 2,73 & 68,19 & 68,19 \\
\hline PC2 & 0,96 & 23,89 & 84,13 & 1,15 & 28,74 & 96,93 \\
\hline PC3 & 0,63 & 15,87 & 100,00 & 0,12 & 3,07 & 100,00 \\
\hline PC4 & 0,00 & 0,00 & 100,00 & 0,00 & 0,00 & 100,00 \\
\hline
\end{tabular}

$\mathrm{PC}=$ principal components.

position in the plane defined by principal component analysis $1,2,3 \ldots$ (PC1, PC2, PC3...) from multivariate statistical analysis. Thus, tPCA was carried out to investigate the roles of different transgenic and nontransgenic soybean samples produced in two different regions, according to Wu et al. (2008).

The eigenvalues (Table 6 ) shows that $100 \%$ of the variance in original data is explained by the first three components. The third principal component has close value to the second component. Therefore, only three components were retained to principal components analyses to the non-transgenic soybean samples produced in both regions. The first component, PC1, explains $60.24 \%$ of the variance, the second component PC2 is associated with $23.89 \%$ and the third component PC3 explains $15.87 \%$ to non-transgenic soybean samples. Already for the transgenic soybean sample, two components were selected to explain about $96.93 \%$ of the total variance, PC1 (68.19\%) and PC2 (28.74\%). The components analysis has been used in analogous studies about soybean samples (Martins, Juliatti, Santos, Polizel, \& Juliatti, 2007).

Fig. 1 shows the principal components analysis (score/samples "part a" and variables/loadings "part b") of fatty acids non-transgenic soybean samples. Analyzing Fig. 1a, we can see two groups formed. The first one was composed for soybean samples produced in Ponta Grossa's region, except the BRS184, BRS262 and BRS268 variety produced in Londrina's region. However, the second one was characterized by samples obtained in Londrina's region except the BRS233, BRS257 and BRS260 varieties produced in Ponta Grossa's region. These exceptions can be seen in the loadings graphics. The first principal component (Fig. 1b) showed that MUFA and PUFA variables are important in explaining the entire set of data. However, the loadings graphics on PC2 and PC3 (Fig. 1b), the SFA and Environment variable are important to explaining the data, respectively. Souza et al. (2007) also used the results of loadings analysis to verify the importance of the variables studied.

The same analyses (Fig. 2) were realized in the transgenic soybean samples produced in Londrina's and Ponta Grossa's region. The score graphics (Fig. 2a) showed positive and negative values on PC1, to BRS246RR, BRS255RR samples obtained Ponta Grossa's region and BRS242RR, BRS244RR and BRS245RR varieties grown in Londrina, respectively. On PC2 also were observed extreme values to BRS242RR, BRS244RR and BRS245RR soybean varieties produced in Ponta Grossa's region and BRS255RR and BRS256RR obtained Londrina's region. On loadings graphics (Fig. 2b) we can observe that MUFA, PUFA and Environment variables are most important than SFA variable on PC1, but on PC2 the SFA and Environment variables were important for explains the set data. These differences in the importance between variables explain the separation of transgenic soybean samples.

Through evaluation of the obtained fatty acid composition tables and data from PCA it can be inferred some conclusions: for non transgenic soybeans the MUFA variable showed negative correlation with PC1 while both environment and PUFA correlated with PC1 in a positive manner. The P1, P2, P4, P7, P10, P12 and L13 varieties possess inferior MUFA amounts, when compared to the remaining fatty acids, and superior PUFA quantities for L3, L4, L5, L6, L8, L9, L10, L12, L14 and P9 varieties.

For transgenic samples, the most important variable for this study were the same as above, but with inverted correlations regarding PC1. In the same manner, it can be expected that the greatest MUFA contents can be extracted from TP3, TP4 and TP5 transgenic varieties. If oils with higher PUFA percentages are the main interest, TL1, TL2, TL3 and TL4 cultivars must be selected.

\section{Conclusions}

The fatty acid analyses showed highest concentration of PUFA in transgenic and non-transgenic soybean samples grown in different regions. Furthermore, also were founded essential fatty acids such as linoleic and linolenic. Beyond that, the exploratory statistical method PCA has been shown to provide a simple, rapid, and effective way to
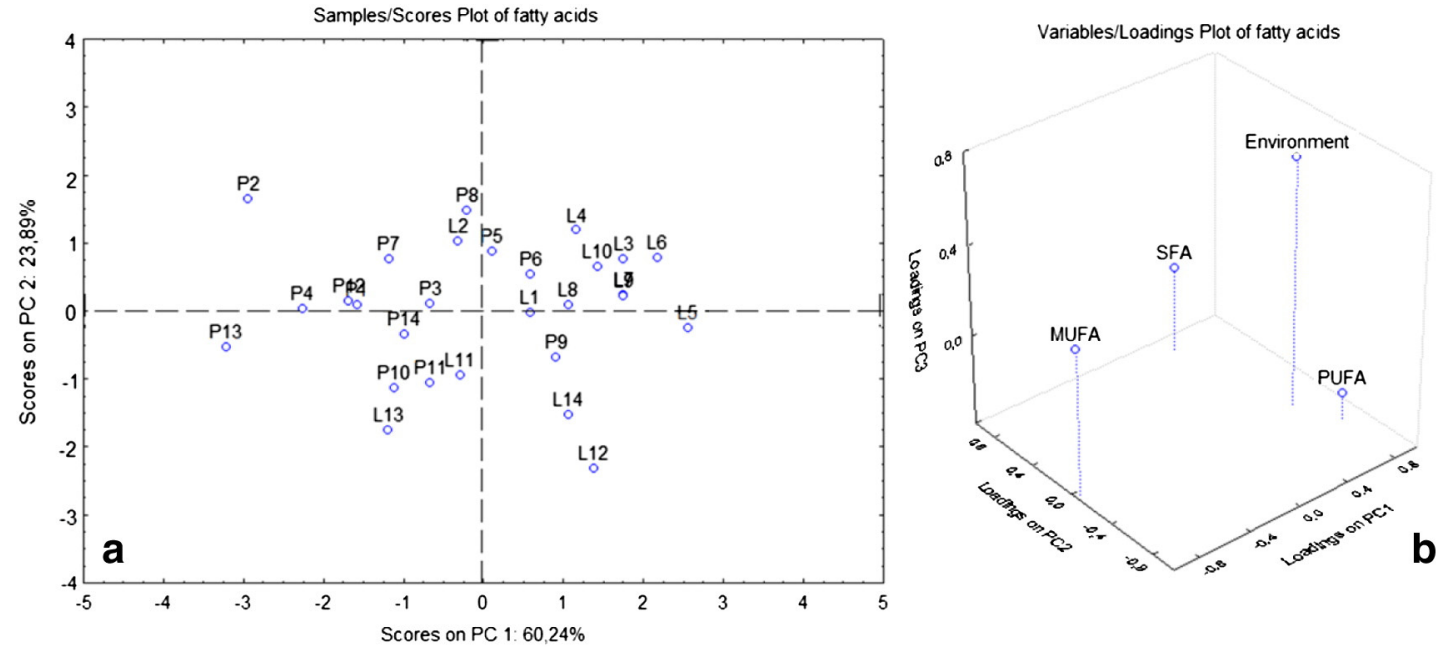

Fig. 1. Scores (a) and loadings (b) plot for the first and second PC of non-transgenic soybean samples obtained from the both regions. 

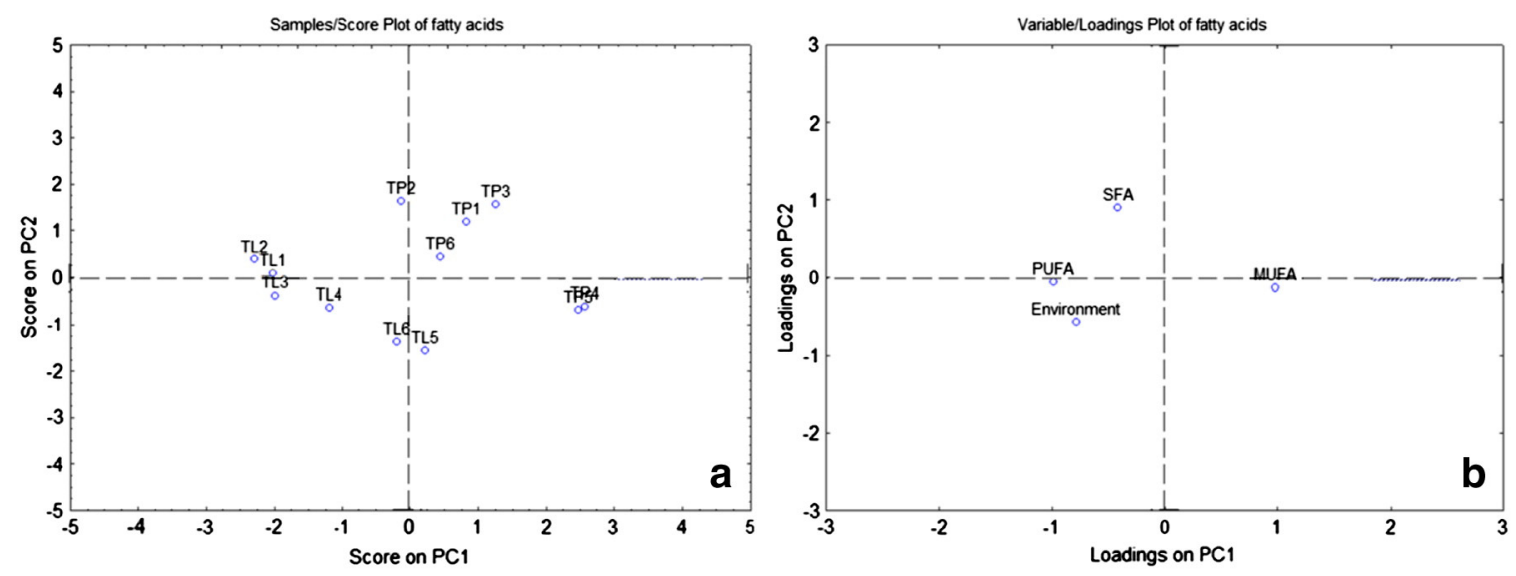

Fig. 2. Scores (a) and loadings (b) plot for the first second PC of transgenic soybean samples obtained from the both regions.

differentiate the transgenic and non-transgenic soybeans grown in different regions. Moreover, this analysis can detect which variable is most important to differentiate the set data.

\section{Acknowledgements}

We thank CAPES for the financial support and EMBRAPA Soja for donation of soybean grains.

\section{References}

Almeida, V. V., Bonafe, E. G., Muniz, E. C., Matsushita, M., Souza, N. E., \& Visentainer, J. V. (2009). Optimization of carrot leaf dehydratation aiming at the preservation of omega-3 fatty acids. Ouimica Nova, 32, 1334-1337.

Berman, K. H., Harrigan, G. G., Riordan, S. G., Nemeth, M.A., Hanson, C., Smith, M., et al. (2010). Compositions of forage and seed from second-generation glyphosatetolerant soybean MON 89788 and insect-protected soybean MON 87701 from Brazil are equivalent to those of conventional soybean (Glycine max). Journal of Agriculture and Food Chemistry, 58, 6270-6276.

Bligh, E. G., \& Dyer, W. J. (1959). A rapid method of total lipid extraction and purification. Canadian Journal of Biochemistry and Physiology, 37, 911-917.

Bonafe, E. G., Aguiar, A.C., Boroski, M., Monteiro, J., Souza, N. E., Matsushita, M., et al. (2011). Quantification of EPA and DHA in seafood of the south coast of Brazil. Nutrition and Food Sciense, 41, 401-411.

Carbonera, F., Bonafe, E. G., Martin, C. A., Montanher, P. F., Ribeiro, R. P., Figueiredo, L. C. et al. (2014). Effect of dietary replacement of sunflower oil with perilla oil on the absolute fatty acid composition in Nile tilapia (GIFT). Food Chemistry, 148, 230-234.

Cheng, K. C., Beaulieu, J., Iquira, E., Belzile, F. J., Fortin, M. G., \& Strömvik, M. V. (2008). Effect of transgenes on global gene expression in soybean is within natural range of variation of conventional cultivars. Journal of Agriculture and Food Chemistry, 56, 3057-3067.

Daniels, R. L., Kim, H. J., \& Min, D. B. (2006). Hydrogenation and interesterification effects on the oxidative stability and melting point of soybean oil. Journal of Agriculture and Food Chemistry, 54, 6011-6015.

Droste, A., Pasquali, G., \& Bodanese-Zanettini, M. H. (2002). Transgenic fertile plants of soybean (Glycine $\max (\mathrm{L}$.) Merrill) obtained from bombarded embryogenic tissue. Euphytica, 127, 367-376.

EMBRAPA (2011). Tecnologias de produção de soja: região central do Brasil 2012 e 2013. Londrina: Embrapa Soja, 261.

International Organization for Standardization (ISO 5509). (1978). Animal and vegetable fats and oils - Preparation of methyl esters of fatty acids. : International Organization for Standardization - ISO, 1-6.

Kumar, V., Rani, A., Solanki, S., \& Hussain, S. M. (2006). Influence of growing environment on the biochemical composition and physical characteristcs of soybean seed. Journal of Food Composition and Analysis, 19, 188-195.

Lee, S. J., Ahn, J. K., Kim, S. H., Kim, J. T., Han, S. J., Jung, M. Y., et al. (2003). Variation in isoflavone of soybean cultivars with location and storage duration. Journal of Agriculture and Food chemistry, 51, 3382-3389.

Lee, S. J., Choi, J. Y., Park, S., Chung, J. I. I., Jin, J. S., Lee, S. J., et al. (2010). Determination of phospholipids in soybean (Glycine max (L.) Merrill) cultivars by liquid chromatography-tandem mass spectrometry. Journal of Food Composition and Analysis, 23, 314-318.
Lee, S. J., Kim, J. J., Moon, H. I., Ahn, J. K., Chun, S.C., Jung, W. S., et al. (2008). Analysis of isoflavones and phenolic compounds in Korean soybean [Glycine max (L.) Merrill] seeds of different seed weights. Journal of Agriculture and Food Chemistry, 56, 2751-2758.

Lerat, S., England, L. S., Vincent, M. L., Pauls, K. P., Swanton, C. J., Klironomos, J. N., et al. (2005). Real-time polymerase chain reaction quantification of the transgenes for roundup ready corn and roundup ready soybean in soil samples. Journal of Agriculture and Food Chemytri, 53, 1337-1342.

Lichtenstein, A., Matthan, N. R., Jalbert, S. M., Resteghini, N. A., Schaefer, E. J., \& Ausman, L. M. (2006). Novel soybean oils with different fatty acid profiles alter cardiovascular disease risk factors in moderately hyperlipidemic subkects. American Journal Clinical Nutrition, 84, 497-504

Maestri, D.M., Labuckas, D. O., Meriles, J. M., Lamarque, A. L., Zygadlo, J. A., \& Guzman, C. A. (1998). Seed composition of soybean cultivars evaluated in different environmental regions. Journal of the Science of Food and Agriculture, 77, 494-498.

Martins, J. A. S., Juliatti, F. C., Santos, V. A., Polizel, A.C., \& Juliatti, F. C. (2007). Latent period and the use of principal components analysis for partial resistance to soybean rust Summa Phytopathologica, 33, 364-371.

Milinski, M. C., Visentainer, J. V., Martin, C. A., Arias, C. A. A., Matsushita, M., \& Souza, N. E. (2007). Proximate composition and fatty acids profile of Brazilian conventional and transgenic soybeans (Glycine $\max (\mathrm{L}$.) Merrill) cultivars. Electronic Journal of Environmental Agricultural and Food Chemistry, 6, 1905-1911.

Nodari, R. O., \& Guerra, M. P. (2003). Plantas transgênicas e seus produtos: impactos, riscos e segurança alimentar (Biossegurança de plantas transgênicas). Revista de Nutrição, 16, 105-116.

Patil, A., Taware, S. P., Oak, M.D., Tamhankar, S. A., \& Rao, V. S. (2007). Improvement of oil quality in soybean [Glycine max (L.) Merrill] by mutation breeding. Journal of the American Chemical Society, 84, 1117-1124.

Ribeiro, A.B., Bonafe, E. G., Silva, B. C., Montanher, P. F., Junior, O. S., Boein, J. S., et al. (2013). Antioxidant capacity, total phenolic content, fatty acids and correlation by principal component analysis of exotic and native fruits from Brazil. Journal of Brazilian Chemical Society, 24, 797-804.

Rott, M. E., Lawrence, T. S., Wall, E. M., \& Green, M. J. (2004). Detection and quantification of roundup ready soy in foods by conventional and real-time polymerase chain reaction. Journal of Agriculture and Food Chemitry, 52, 5223-5232.

Souza, P. P., Siebald, H. G. L., Augusti, D.V., Neto, W. B., Amorim, V. M., Catharino, R. R., et al (2007). Electrospray ionization mass spectrometry fingerprinting of Brazilian artisan cachaça aged in different wood casks. Journal of Agriculture and Food Chemistry, 55, 2094-2102.

Souza, L. C. F., Zanon, G. D., Pedroso, F. F., \& Andrade, L. H. L. (2009). Teor de proteína e de óleo nos grãos de soja em função do tratamento de sements e aplicação de micronutrientes. Ciência e Agrotecnologia, 33, 1586-1593.

Tanamati, A., Stevanato, F. B., Visentainer, J. E. L., Matsushita, M., Souza, N. E., \& Visentainer, J. V. (2009). Fatty acid composition in wild and cultivated pacu and pintado fish. European Journal of Lipid Sciense and Technology, 111, 183-187.

Wu, Z. G., Rodgers, R. P., \& Marshall, A. G. (2004). Characterization of vegetable oils: Detailed compositional fingerprints derived from electrospray ionization Fourier transform ion cyclotron ressonance mass spectrometry. Journal of Agriculture and Food Chemistry, 52, 5322-5328.

Wu, W., Zhang, Q., Zhu, Y., Lam, H. M., Cai, Z., \& Guo, D. (2008). Comparative metabolic profiling reveals secondary metabolites correlated with soybean salt tolerance. Journal of Agriculture and Food Chemistry, 56, 11132-11138.

Zobiole, L. H. S., Oliveira, R. S., Jr., Visentainer, J. V., Kremer, R. J., Bellaloui, N., \& Yamada, T. (2010). Glyphosate affects seed composition in glyphosate-resistant soybean. Journal of Agriculture and Food Chemitry, 58, 4517-4522. 